Áreas Naturales Protegidas Scripta, 2016. Vol. 2 (2): 67-89. https://doi.org/10.18242/anpscripta.2016.02.02.02.0004

Human population, socio-economic activities and social-environmental problems of Protected Natural Areas of Mexico

\title{
Población humana, actividades socioeconómicas y problemáticas socioambientales de las Áreas Naturales Protegidas (ANP) de México
}

\author{
Magdalena Lagunas-Vázques ${ }^{1 *}$, Luis Felipe Beltrán-Morales ${ }^{1}$, \\ Mariana Bobadilla-Jiménez ${ }^{1}$ y Alfredo Ortega-Rubio ${ }^{1}$
}

\section{Abstract:}

This work includes a descriptive review of the basic social aspects of a protected natural area (PNA) and of the human populations settled in the PNAs in the country where approximately $3.9 \%$ of the Mexican population lives in them. The main socioeconomic activities developed by the inhabitants of PNAs and their most representative socioenvironmental issues are categorized. Just over $80 \%$ of the communities located within the geographical limits of the PNAs are populated with less than 15,000 people. This eminent rurality determines the current and potential conditions, especially the actions of rural development and resource management in all PNAs of Mexico by local residents. Undoubtedly, the solution of the socioenvironmental problems described, as well as improving life quality of local inhabitants, must be achieved through local actions of rural sustainable development.

Key words: Protected Natural Areas, human population, local inhabitants, indigenous, socioeconomic activities, socioenvironmental problems, rurality.

\section{Resumen}

El presente trabajo incluye una descripción de los aspectos sociales básicos de las ANP: se hace una revisión de la población humana de las ANP del país. Aproximadamente el 3.9\% de la

\footnotetext{
${ }^{1}$ Centro de Investigaciones Biológicas del Noroeste S.C. La Paz, B.C.S., 230906, México.
}

*Autora correspondencia: mlagunas@cibnor.mx 
población mexicana vive en una ANP. Se categorizan las principales actividades socioeconómicas que desarrollan los habitantes de las ANP, así como sus problemáticas socioambientales más representativas. Poco más del $80 \%$ de los asentamientos que se encuentran dentro de los límites geográficos de las ANP son localidades con poblaciones menores a los 15,000 habitantes. Esta eminente ruralidad determina las condiciones actuales y las potenciales, especialmente en cuanto a las acciones de desarrollo rural y manejo de recursos en las ANP de México, por parte de sus pobladores locales. Indudablemente, la resolución de las problemáticas socioambientales descritas

y el incremento de los niveles de vida de los pobladores locales de las ANP de México deben de transitar por soluciones locales de desarrollo sustentable rural.

Palabras clave: Áreas Naturales Protegidas, población humana, habitantes locales, indígenas, actividades socioeconómicas, problemáticas socioambientales, ruralidad.

\section{Antecedentes}

Las Áreas Naturales Protegidas son una de las principales herramientas utilizadas para propiciar la conservación de la naturaleza tanto alrededor del mundo (Duran y Jiménez, 2010; WCPA, 2016), como en nuestro país (Bezaury-Creel y Gutiérrez-Carbonell, 2009). En México antes de que la gran mayoría de las ANP fueran declaradas como tales ya tenían grupos humanos habitándolas y haciendo uso de sus recursos naturales (Bezaury-Creel y Gutiérrez-Carbonell, 2009). En Centro y Sur América poco más del 80\% de sus ANP están habitadas por pueblos indígenas (Alrcon, 1994). Alcorn establece que gran parte de la biodiversidad del planeta se encuentra en territorios indígenas, situación que también ocurre en el caso de México según lo expuesto por Boege (2008, 2009).

Existe un traslape muy claro entre las áreas de mayor biodiversidad del planeta y las regiones densamente habitadas por población indígena del mundo, haciendo coincidir las porciones de alta diversidad biológica y lingüística (Toledo, 2001, 2005; Maffi, 2005). De acuerdo con el Fondo Mundial para la Conservación de la Naturaleza (WWF siglas en inglés) (2000), en el 94\% de las 238 eco-regiones terrestres reconocidas como las más importantes por su diversidad biológica en el mundo se encuentran 4,635 grupos etnolingüísticos; esa cifra representa el $67 \%$ de todos los grupos etnolingüísticos identificados en el mundo. 
Los acuerdos internacionales en temas sobre conservación biológica y desarrollo sostenible consideran en primer orden de importancia la participación en las prácticas de manejo, la interacción y la dependencia de los ecosistemas naturales por parte de las comunidades indígenas y locales en el mundo (Maffi, 2005). Así, el Capítulo 26 de la Agenda 21, como el Convenio sobre la Diversidad Biológica (1992) reconocen y fortalecen el papel de las poblaciones indígenas y sus comunidades y sus estilos tradicionales de vida en pro de la conservación y la utilización sostenible de la diversidad biológica.

Berkes et al (2000), consideran que existe una co-evolución entre cultura y naturaleza; y de acuerdo a Pretty et al (2008) esta interacción es un proceso que se ha venido dando desde hace miles de generaciones; por lo que la conservación de la biodiversidad es imposible sin tomar en cuenta el conjunto de factores sociales que la condicionan (Mascia et al., 2003). De acuerdo a lo planteado por Davidson-Hunt et al. (2016) es imprescindible conocer y valuar la diversidad de interrelaciones entre la gente de las comunidades rurales remotas y la naturaleza para promover la continuidad de los conocimientos existentes y generar nuevos conocimientos (resiliencia) sobre las relaciones entre los seres humanos y la naturaleza. Muchas sociedades tradicionales rurales que coexisten con la naturaleza adyacente y dependen de ella para su sobrevivencia implementan convivencias que permiten la conservación de la biodiversidad (MMBT., 2004; Pretty et al., 2008).

Durante las últimas décadas, a nivel mundial, se ha generado un conjunto considerable de información que respalda el conocimiento ecológico tradicional (Traditional Ecological Knowledge) y los saberes locales en el uso y manejo de los recursos naturales por parte de los habitantes locales de los entornos rurales, incluyendo la concepción del término sistema socioecológico (Ostrom, 1991; Chambers y Conway, 1991; Berkes, 2001; Toledo 2001, 2005; Pretty et al., 2008; Merino y Martínez, 2013).

En un minucioso estudio Boege (2009) analizó la coincidencia geográfica de los territorios con presencia mayoritaria de población indígena con las regiones de mayor importancia en diversidad biológica de México propuestas por la Comisión Nacional para el Conocimiento y Uso de la Biodiversidad (CONABIO). Al aplicar los criterios CONABIO encontró que la mayoría de pueblos indígenas se encuentra en ellas, con más de 10, 786,914 hectáreas. Esto representa 7\% del total de sus áreas y $26.3 \%$ de la superficie de ANP.

De acuerdo con Boege (2009) los indígenas han interactuado en procesos de larga duración con 
los ecosistemas de muy alta diversidad biológica, reconocidos como Centros de diversificación biocultural (biocultural hotspots), que son los territorios que coinciden con los centros de origen y diversificación tanto de la biodiversidad natural así como de la diversidad domesticada con los territorios de los pueblos (Boege, 2008). Trabajando en esta misma línea Toledo (2007) ha generado una propuesta Bio-Regional, para el país, que incluye conservación natural y cultural, la cual comprende tres axiomas: 1. Axioma bio-social, 2. Axioma bio-cultural, y 3. Axioma bioproductivo.

México cuenta con un rasgo fundamental que se requiere abordar para tratar temas de conservación, biodiversidad y desarrollo rural: más de la mitad de su superficie está distribuida entre más de 30,000 ejidos y comunidades rurales, 77\% de los cuales tienen superficies menores a 3,000 hectáreas, y poco más del 50\% de estos núcleos agrarios poseen al menos cada uno de ellos 200 hectáreas de ecosistemas de bosque, selva o matorral forestal (Reyes et al., 2012). De acuerdo a un estudio nacional liderado por el Registro Agrario Nacional (RAN) el país está conformado por un mosaico de unidades territoriales rurales con una alta correlación entre la propiedad social y la distribución de los recursos naturales (Reyes et al., 2012).

De acuerdo a Merino y Martínez (2013) México tiene la mayor extensión de bosques y selvas manejada por comunidades en el mundo (el 18\% de los bosques son manejados por comunidades locales), los bosques comunitarios aportan $80 \%$ de la madera que se produce a nivel nacional. Según las mismas autoras una tercera parte de las comunidades analizadas en su estudio cuenta con un Plan de Manejo Forestal, 15\% procesan la madera agregándole valor, el 46\% de las comunidades han creado áreas dedicadas exclusivamente a la protección de los sistemas forestales. Muchas de estas comunidades han invertido ganancias en generar bienes y servicios públicos en los pueblos que habitan, en general tal como lo mencionan las autoras México tiene una extensa experiencia en el manejo de sus bosques comunitarios (Merino y Martínez, 2013).

El objetivo principal del trabajo es hacer una descripción de los aspectos sociales básicos de las ANP del país, cuantificando la población humana de las ANP, identificando la población indígena. Asimismo se ubican los entornos rurales de las ANP. Categorizando las principales actividades socioeconómicas que desarrollan dichos habitantes de las ANP, así como sus problemáticas socioambientales más representativas.

El término rural en ciencias sociales tradicionalmente se ha venido utilizando como lo opuesto 
a lo urbano, reconocido en el siglo pasado como el concepto más tajante de diferenciación sobre la división del trabajo (rural/urbano) (García-Bartolomé, 1991). Otra variante para diferenciar lo urbano de lo rural es la categoría censal de número de habitantes (Dirven, 2007). En México el criterio censal que se utiliza a través del Instituto Nacional de Estadística y Geografía (INEGI) es el siguiente: una localidad rural es aquella donde habitan 2,500 habitantes (Dirven, 2007).

Para los fines del presente análisis se tomó básicamente el tamaño de la población para definir el entorno rural del ANP, hemos considerado lo rural por entorno (donde a veces se incluyen más de una localidad medianamente cercanas entre sí), que comparten ecosistemas, es decir se encuentran en una misma ANP; por lo tanto en lugares donde confluyen más de una localidad o exista una localidad con una población de hasta 15,000 habitantes se considerara entorno rural dentro de este trabajo, por obvias razones el entorno rural en este trabajo será un espacio geográfico no plenamente georreferenciado.

La información sobre la población humana se tomó de las bases de datos que ofrece la Comisión Nacional de Áreas Naturales Protegidas CONANP en línea, vertida en las fichas técnicas de cada ANP decretada (http://www.conanp.gob.mx/que_hacemos/. Link principal y a partir de dicha dirección electrónica se procedió a consultar individualmente cada ficha técnica de cada ANP por categoría, por lo que resulta imposible poner aquí desplegada la liga para cada ANP consultada). Es importante mencionar que de 177 ANP decretadas en el país, para 71 ANP no existen datos o no tienen población humana (pues las bases de datos de la CONANP no son específicas), eso corresponde aproximadamente al 40\% de las ANP. De las 41 Reservas de la Biosfera (RB) decretadas nueve no tienen datos de población humana. De 66 Parques Nacionales (PN) 37 no tienen datos de población humana. De los cinco Monumentos Naturales (MN) solo tres tienen datos sobre la población humana. De las 8 Áreas de Protección de Recursos Naturales (APRN) en una no hay datos población. De las 39 Áreas de Protección de Flora y Fauna (APFF) en 6 no hay datos sobre habitantes humanos. En los 18 Santuarios (S) no hay datos de población en 16 de ellos.

Para clasificar las actividades socioeconómicas se consideró la definición del Instituto Nacional de Estadística y Geografía (INEGI, 2016), donde: Actividades económicas: Son las actividades cuyo propósito fundamental es producir bienes y/o servicios. Las actividades económicas se pueden clasificar en distintos niveles de desagregación según el tipo de bien o servicio.

Para la clasificación de problemática ambiental en este estudio se consideraron las definiciones 
sobre daño ambiental e impacto ambiental vertidas en el Artículo 3. Del Reglamento en materia de impacto ambiental de la Ley General de Equilibrio Ecológico y Protección al Ambiente vigente. Que contiene las siguientes definiciones. III.- Daño ambiental: Es el que ocurre sobre algún elemento ambiental a consecuencia de un impacto ambiental adverso; IX. Impacto ambiental significativo o relevante: Aquel que resulta de la acción del hombre o de la naturaleza, que provoca alteraciones en los ecosistemas y sus recursos naturales o en la salud, obstaculizando la existencia y desarrollo del hombre y de los demás seres vivos, así como la continuidad de los procesos naturales.

Para identificar las principales actividades socioeconómicas y problemáticas socioambientales, se consultó la información vertida en el portal de la CONANP, específicamente de todas y cada una de las fichas técnicas para cada ANP decretada. Se utilizó la lista de actividades socioeconómicas y problemáticas socioambientales que contienen las fichas, haciendo una base de datos, posteriormente. Se organizó y clasificó la información con criterios relacionales de acuerdo a las definiciones utilizadas en INEGI (2016) para actividades socioeconómicas y en la legislación ambiental para problemática ambiental: agrupándose en el caso de las actividades socioeconómicas sin considerar su magnitud ni continuidad en el tiempo, las actividades fueron consideradas por el solo hecho de estar en la lista de las fichas técnicas de cada ANP (tomando de forma invariable las palabras tal como están redactadas en las fichas, es decir cómo fueron nombradas por las personas que proporcionaron dicha información, es decir desde la dirección administrativa de cada ANP), al final se procesó la información con estadística descriptiva y se elaboraron histogramas de frecuencia por actividad y problemática, así como por ANP y por cada una de las seis categorías (RB, PN, MN, APRN, APFF, S).

\section{La población humana de las ANP de México}

De acuerdo a la información analizada aproximadamente el 3.9\% de la población mexicana vive en un ANP (Para detalles sobre la población en cada ANP ver el Anexo 1). En México de 4, 689, 557 de habitantes de las ANP, el 5.7\% de ellos corresponde a población indígena, es decir aproximadamente 270, 476 personas (Tabla 1). Del total de ANP del país hasta abril del 2016 un $46 \%$ de ellas albergan población indígena. Del total de la superficie de terreno que conforma a las ANP del país que son 25, 628,238 hectáreas CONANP (2016), más del 60\% de ésta superficie es de propiedad social (Bezaury-Creel y Gutiérrez-Carbonell, 2009), incluyendo en este tipo de propiedad más de un 30\% de territorio indígena (Boege, 2009). 
Tabla 1. Población humana por categoría en las ANP del país

\begin{tabular}{cccc}
\hline Categoría & Población total & $\begin{array}{c}\text { Población Indígena y de la } \\
\text { población total }\end{array}$ & $\begin{array}{c}\text { \% población por } \\
\text { categoria }\end{array}$ \\
\hline RB & 438263 & $64917(15 \%)$ & $9 \%$ \\
PN & 3544296 & $79716(2 \%)$ & $76 \%$ \\
MN & 168 & $63(38 \%)$ & $0.004 \%$ \\
APRN & 408009 & $109811(27 \%)$ & $9 \%$ \\
APFF & 298737 & $15969(5 \%)$ & $6 \%$ \\
S & 84 & & $0.002 \%$ \\
\hline Totales & 4689557 & $270476(6 \%)$ & $100 \%$ \\
\hline
\end{tabular}

Fuente: Elaboración propia con datos tomados de la CONANP (2016)

Figura 1a. Proporción de la población concentrada por cantidad de habitantes en RB

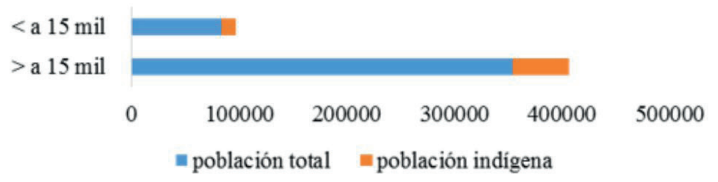

Figura 1b. RB con localidades de más de 15 mil habitantes

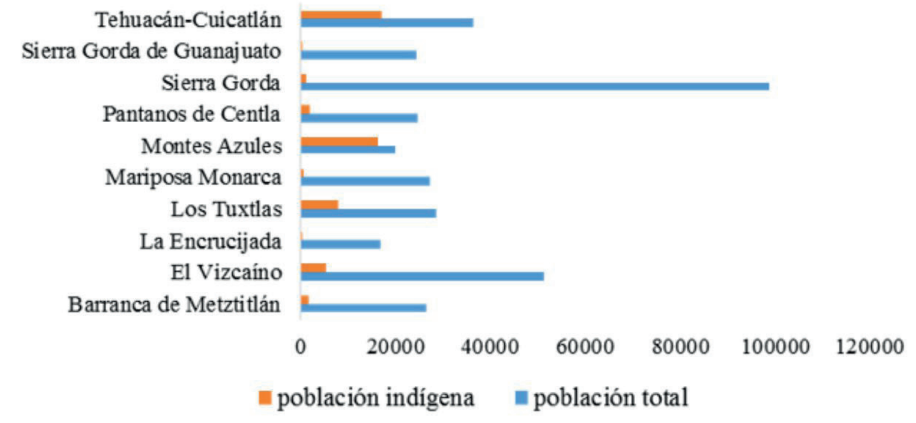

Figura 1c. Proporción de la población por tamaño de la localidad en $\mathrm{RB}$ y Proporción de población total e indígena en $\mathrm{RB}$

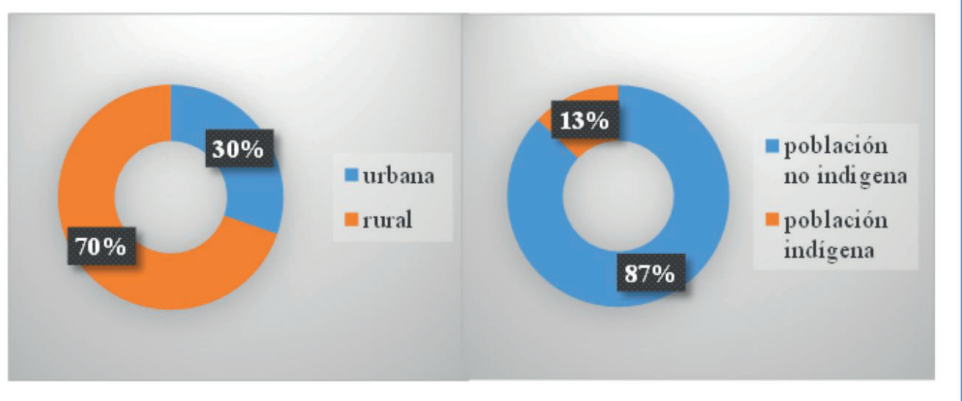

Fuente: Elaboración propia con datos tomados de la CONANP (2016)

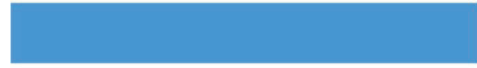

FIGURA 1: RESERVAS DE LA BIOSFERA

Las $\mathrm{RB}$ representan un $23 \%$ de las ANP del país, poseen un $9 \%$ de la población de las ANP. Es

la categoría con mayor territorio protegido 12751 149 hectáreas repartido en 41 ANP, incluyen a 438 263 habitantes, presentan 11 localidades con población superior a los 15 mil habitantes (Figuras 1a y 1b), sin embargo la mayoría de las $\mathrm{RB}$ presentan un ambiente rural (Figura 1c). Entre sus habitantes el $13 \%$ son indígenas. 
Figura 2a. Porcentaje de la población concentrada por cantidad de habitantes en PN

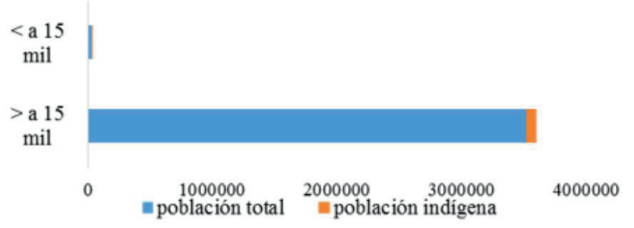

Figura 2b. PN con localidades de más de 15 mil habitantes

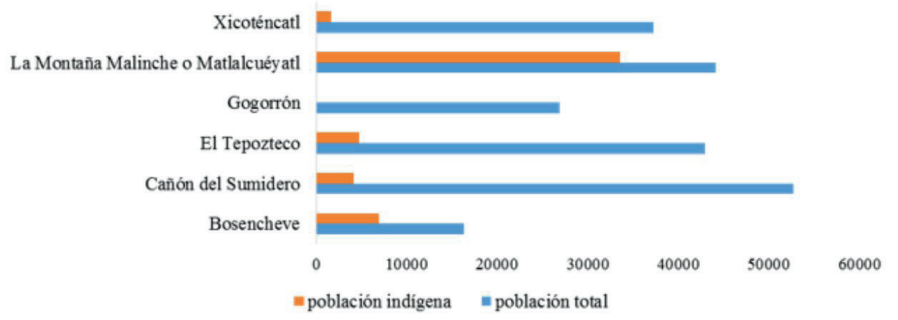

Figura 2c. Proporción de la población por tamaño de la localidad en PN y Proporción de población total e indígena en PN

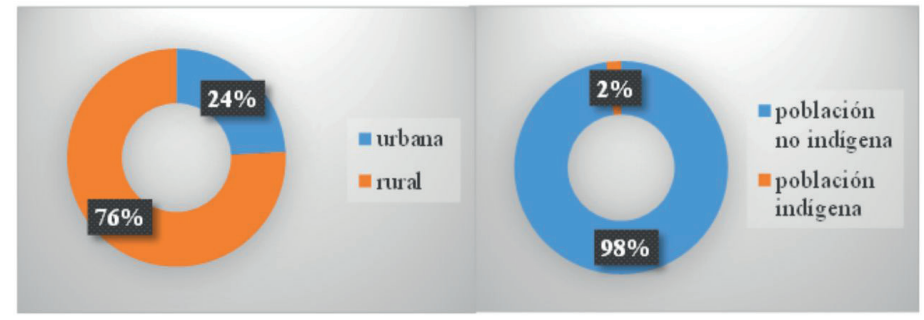

Fuente: Elaboración propia con datos tomados de la CONANP (2016)

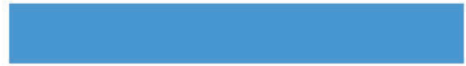

FIGURA 2. PARQUES NACIONALES

Los PN representan un

$37 \%$ de las ANP del país

(son 66 PN en total),

poseen un $76 \%$ de la

población humana de las

ANP. Son la categoría con

mayor número de

habitantes 3544 296, seis

PN presentan poblaciones

superiores a los $15 \mathrm{mil}$

habitantes (Figuras 2a y

2b), el PN Cañón de Río

Blanco no se consideró en

la figura porque él solo

$\begin{array}{llll}\text { posee } & 3 & 297 & 999\end{array}$

habitantes, por lo que se

salía de contexto en la

misma. Este PN se ubica

dentro de 11 municipios y

dos Regiones CONANP.

Sin embargo la mayoría de

los PN presentan un

entorno rural (Figura 2c).

Entre sus habitantes el 2\%

son indígenas.

\section{Monumentos Naturales y Santuarios}

Los monumentos naturales son cinco en todo el país, los cuales por su naturaleza poseen muy poca población humana, pues suelen ser recintos muy puntuales, en algunos casos sitios arqueológicos. Es interesante notar por su importancia cultural, que algunos de ellos cuentan con población indígena (Tabla 2). 
Tabla 2. Monumentos naturales y su población

\begin{tabular}{ccc}
\hline Monumento Natural & Población total & Población indígena \\
\hline Bonampak & 11 & 11 \\
$\begin{array}{c}\text { Cerro de la Silla } \\
\text { Río Bravo del Norte } \\
\text { Yagul }\end{array}$ & 8 & \\
Yaxchilán & 149 & 52 \\
& 168 & 63 \\
\hline
\end{tabular}

Fuente: Elaboración propia con datos tomados de la CONANP (2016)

Figura 3a. Proporción de la población concentrada por cantidad de habitantes en APRN

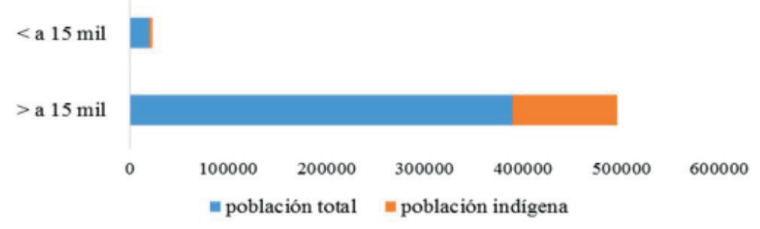

Figura 3b. APRN con localidades de más de 15 mil habitantes

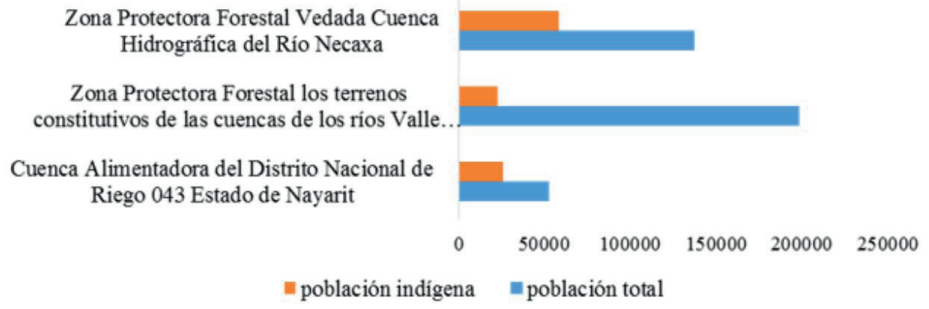

Figura 3c. Proporción de la población por tamaño de la localidad en APRN y Proporción de población total e indígena en APRN

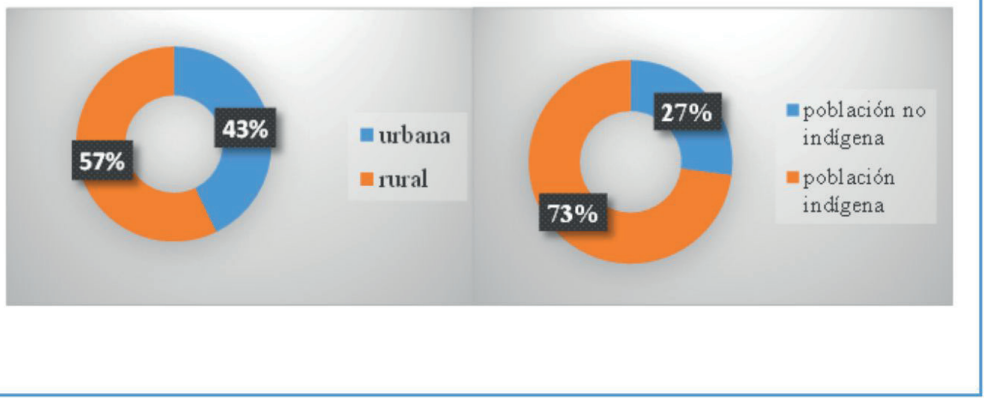

Fuente: Elaboración propia con datos tomados de la CONANP (2016)

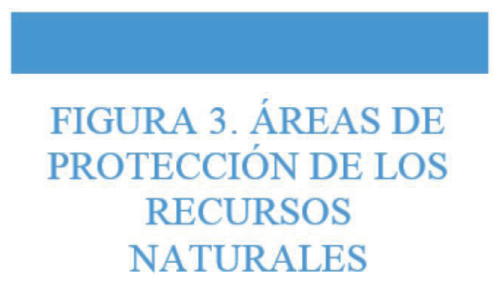

Las APRN representan un

$5 \%$ de las ANP del país (son 8 APRN en total), poseen un $9 \%$ de la población humana de las ANP. Es una de las categorías que posee más población indígena. Más del $50 \%$ de su entorno es rural (Figuras 3a, 3b y 3c).

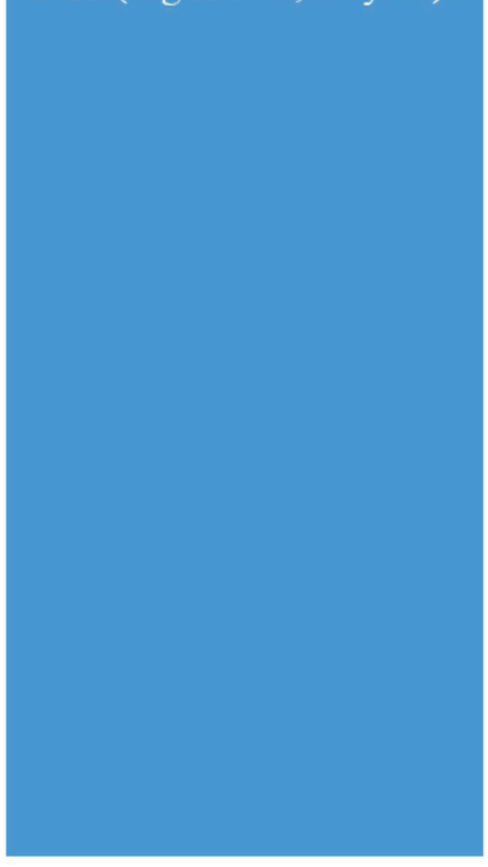


Figura 4a. Proporción de la población concentrada por cantidad de habitantes en APFF

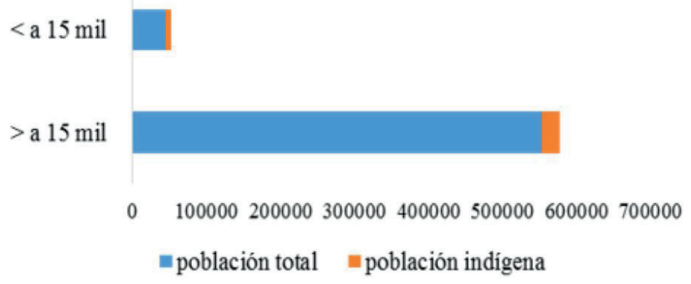

Figura 4b. APFF con localidades de más de 15 mil habitantes

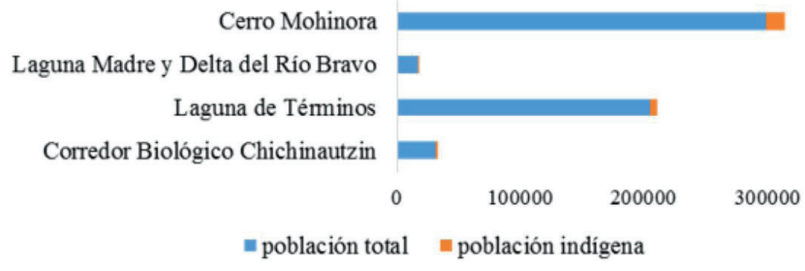

Figura 4c. Proporción de la población por tamaño de la localidad en APFF y Proporción de población total e indígena en APFF

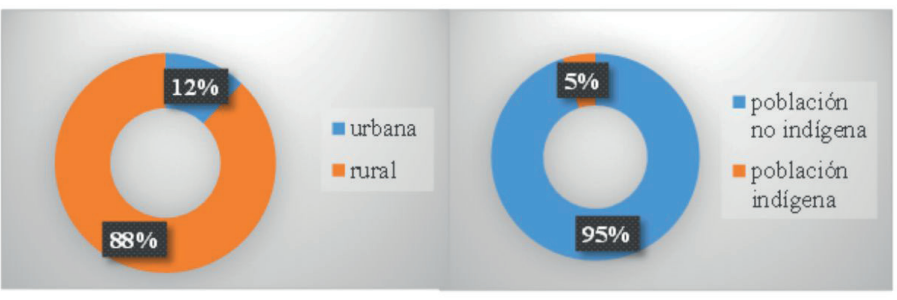

FIGURA 4. ÁREAS DE

PROTECCIÓN DE

FLORA Y FAUNA

Las APFF representan un

$22 \%$ de las ANP del país

(son 39 APFF en total),

poseen un $6 \%$ de la

población humana de las

ANP. Esta categoría

presenta una alta

concentración de

población urbana en pocas

localidades (Figuras 4a y

4b); sin embargo presenta

muchas localidades de

pocos habitantes lo que la

hace que presente un

predominio de su entorno

de tipo rural (Figura 4c),

también posee muy poca

población indígena.

Los Santuarios, representan el 10\% de las áreas protegidas del país, en total son 18, solamente dos tienen población humana y estas son: 1. Islas La Pajarera, Cocinas, Mamut, Colorada, San Pedro, San Agustín, San Andrés y Negrita, y 2. los islotes Los Anegados, Novillas, Mosca y Submarinos y Playa de Puerto Arista con 15 y 69 habitantes rurales respectivamente. 


\section{Principales actividades socioeconómicas y problemáticas socioambientales}

\section{por frecuencia de presencia en las fichas técnicas de todas las ANP del país}

En la Figura 5 se observan las principales actividades y problemáticas por frecuencia de presencia en todas las ANP del país sin tomar en cuenta la categoría de ANP a la que pertenecen. En cuanto a las actividades económicas el desarrollo urbano y cambio de uso de suelo predomina en las ANP del país en una gran proporción, la ganadería extensiva es otra actividad muy representada, y por último en menor grado la infraestructura turística de alto impacto (en este estudio turismo de alto impacto es considerado como equivalente a la definición de turismo de masas, Rábago y Revah, 2014) (Figura 5b). En cuanto a las principales problemáticas más comunes, es decir cacería y/o pesca ilegal y furtiva, contaminacion de agua y suelo e incendios forestales ocurren casi con la misma frecuencia (Figura 6 b).

En la Figura 6 se puede notar que existe una proporción casi equitativa en la presentación de actividades y problemáticas, y que en estas se repiten dos actividades y dos problemáticas, tanto en la presencia global de las ANP del país, como en las más presentes en las seis categorías. Dichas actividades son: socioeconómicas infraestructura turística de alto impacto y ganadería extensiva; y en problemáticas cacería/tráfico ilegal de especies e incendios forestales

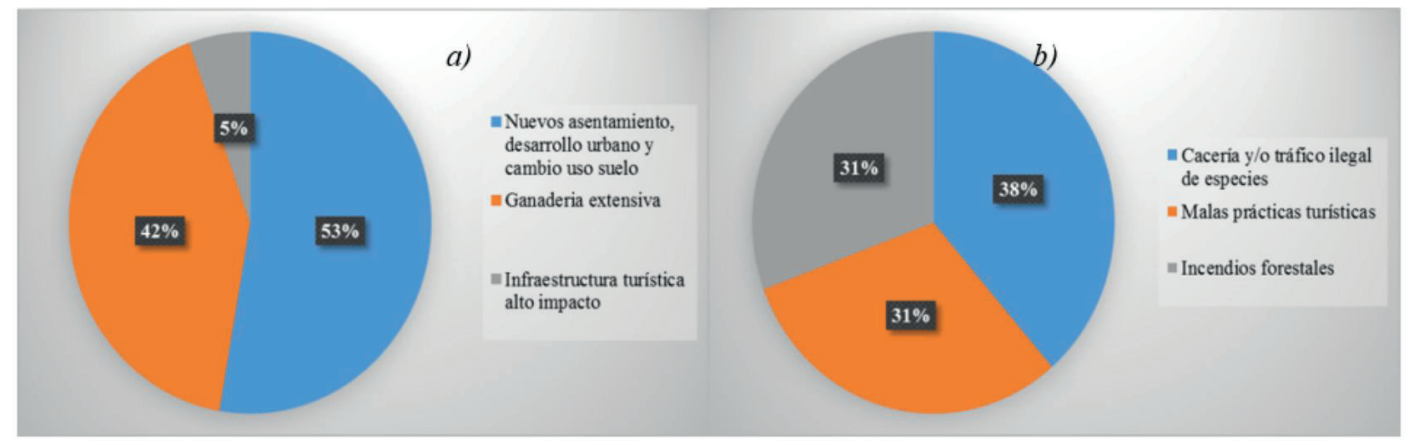

Figura 5. Las actividades socioeconómicas (a) y las problemáticas socioambientales $(b)$ más presentes globalmente en todas las ANP del país sin importar categoría a la que pertenecen.

Fuente: Elaboración propia con datos tomados de la CONANP (2016)

Por último en las Figuras del Anexo 2 se concentraron las 13 actividades socioeconómicas y las 13 problemáticas socioambientales más presentes en todas las fichas técnicas de las ANP del país, el principal objetivo de presentar estas figuras es dar un panorama más completo sobre lo que acontece en las ANP a nivel nacional. En dichas figuras se observan las actividades socioeconómicas y se nota que las más frecuentes tienen que ver con 1) el ramo de la infraestructura y desarrollo 
urbano (actividades tales como nuevos asentamientos, cambio de uso de suelo, infraestructura turística de alto impacto, construcción de autopistas). Otro rubro representativo serían las actividades 2) agrícolas y ganaderas, incluyendo aquí la explotación forestal. Como tercer ramo de importancia por su repetitividad seria la 3) extracción de materiales y la minería. En esos tres grandes grupos de actividades socioeconómicas se pueden condensar las principales actividades de las poblaciones humanas de las ANP de México.

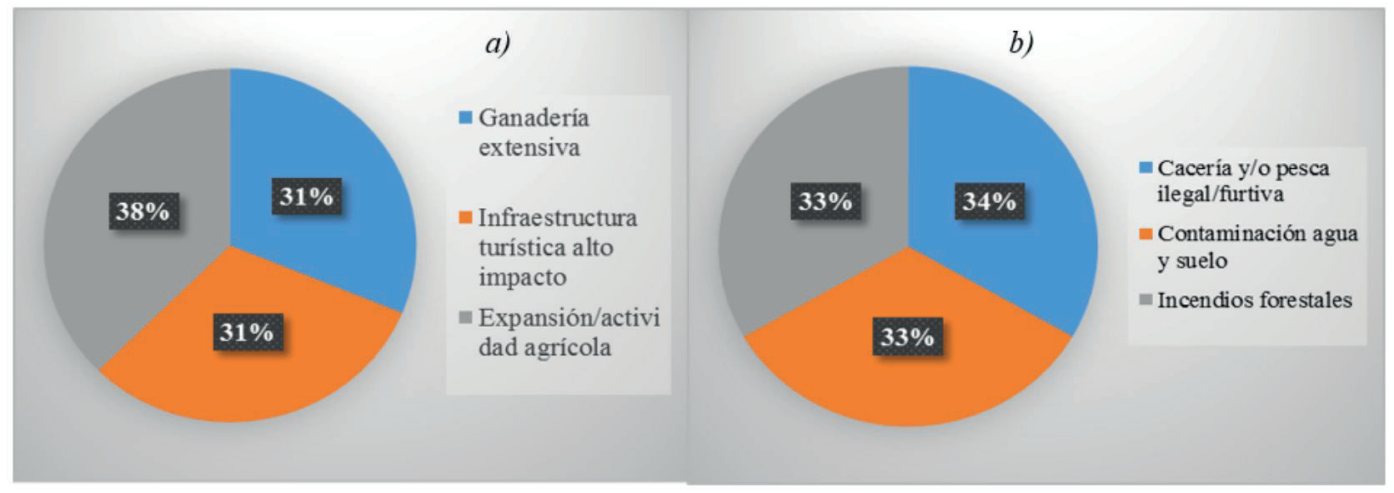

Figura 6. Las actividades socioeconómicas (a) y las problemáticas socioambientales $(b)$ más presentes en las 6 categorías de ANP: RB, PN, MN, APRN, APFF y S.

Fuente: Elaboración propia con datos tomados de la CONANP (2016)

Las problemáticas socioambientales más frecuentes en todas las ANP del país son preocupantes para la conservación de la biodiversidad y la integridad de la naturaleza, básicamente se conjuntan en dos grandes grupos (Anexo 2): 1) contaminación (suelo y agua), erosión, malas prácticas turísticas e incendios forestales y 2) tráfico ilegal de especies (tala clandestina), caza y pesca ilegal e introducción de especies exóticas.

\section{Discusión Académica}

Las ANP del país son instrumentos de conservación de la política ambiental mexicana, dichas ANP se podrían considerar reservas vitales para la vida en general, y por supuesto, la contención humana. Sociopolíticamente hablando son los entornos marco para la sostenibilidad. Es indispensable ubicar qué situaciones y actividades sociales, económicas y políticas se están llevando a cabo en las ANP (Yang et al., 2015), con el fin de reconocer y/o propiciar aquellas actividades más solidarias, equitativas, saludables, es decir: más sostenibles (Bennett y Dearden, 2014a; Bennett y Dearden, 2014b). 
Las principales actividades socioeconómicas que ocurren con mayor frecuencia en las ANP del país son el desarrollo de infraestructura turística de alto impacto, y la ganadería extensiva. Asimismo las principales problemáticas socioambientales que ocurren con mayor frecuencia en las ANP del país son la Cacería/tráfico ilegal de especies y los Incendios forestales.

Considerando que las listas donde se registraron estas actividades y problemáticas fueron expuestas en cada ficha técnica de cada ANP, y que dichas fichas han sido descritas por personas que están en cada una de las direcciones administrativas de cada ANP a lo largo y ancho del país, y que interactúan con el día a día en la dinámica sociocultural-ambiental de sus lugares; estas entidades discursivas, que serían las cuatro palabras-frases arriba enumeradas pueden ser un marco de referencia para abocar esfuerzos y recursos para subsanar y/o mejorar en estos rubros, o para indagar más sobre los mismos y sus situaciones locales.

En este mismo orden de los tres grandes grupos de actividades socioeconómicas en los que se condensan las principales actividades de las poblaciones humanas de las ANP de México: 1. Infraestructura y desarrollo urbano, 2. actividades agropecuarias y 3. Minería. Sería importante que los futuros trabajos de planificación y desarrollo sean compatibles, consensuados y reflexionados en todos los niveles de organización y gobierno (sociedad civil, comunidad, delegación, municipio, estado y federal). Asimismo es importante organizar el territorio de manera armoniosa, pensando en lo que la gente va a hacer, lo que puede hacer, y como quiere vivir. En este sentido es interesante conocer y profundizar en apegarse a lo establecido en Carta Mundial por el Derecho a la Ciudad del programa ONU-Habitat (que no es exclusiva de un entorno urbano).

Sobre las problemáticas socioambientales más frecuentes arriba enunciadas, las estrategias implementadas hasta la fecha no han logrado disminuirlas (Bobadilla-Jiménez et al. 2011), mucho menos erradicarlas, al parecer es indispensable emprender acciones diferentes a las que se han venido haciendo. Se presupone que estas problemáticas están relacionadas con malas prácticas, conductas y hábitos; y que pueden interactuar con las condiciones de pobreza y violencia que abarcan a todo el país. Por lo que las estrategias a implementar tienen que tener un fuerte componente de concientización, además de ser integrales y transversales.

Poco más del $80 \%$ de las localidades que se encuentran dentro de los límites geográficos de las ANP albergan poblaciones menores a los 15,000 habitantes, a pesar de que ciertas ANP presentan conglomeraciones de localidades o una sola localidad con cientos de miles de habitantes. El hecho 
de que un porcentaje mayor de ANP este poco poblada otorga una característica rural eminente a las ANP del país. Por lo mismo esta ruralidad determina las condiciones actuales y las potenciales, especialmente en cuanto a las acciones de desarrollo rural y manejo de recursos en las ANPs de México.

El 40\% de ANP decretadas registradas en la CONANP no tienen datos sobre su población humana, probablemente ello obedece a que no tengan habitantes humanos, como es el caso de los Santuarios. Pero en las bases de datos de la CONANP, no se especifica si esta carencia de los datos de población humana es por ausencia de población o por falta de censos. En virtud de las principales actividades socioeconómicas y problemáticas socioambientales que este trabajo identifica, se recomienda ampliamente la planificación y organización del territorio para realmente incidir en la conservación y el desarrollo sustentable. Asimismo se recomienda un mayor énfasis en promover el cambio en las estrategias estatales de conservación pues la resolución de las problemáticas más frecuentes necesariamente transitan por acciones locales, tales como los aspectos de concientización y cambio de conductas, así como con las situaciones de violencia y pobreza que son comunes en estas localidades. Indudablemente, la alianza con grupos humanos locales y su respectiva concientización es una componente importante a considerar en la conservación de la naturaleza (Chambers y Conway, 1991).

La real integración de las poblaciones locales, campesinas e indígenas, es esencial y requerida para el manejo y conservación de la naturaleza, por la estrecha simbiosis que se da entre los habitantes locales y sus recursos naturales. Al respecto se han documentado diferentes experiencias y perspectivas, desde hace varias décadas, por especialistas de diversas disciplinas que coinciden en temas de comunidades originarias, grupos humanos rurales diversos y desarrollo rural o manejo de recursos, así como conservación de la naturaleza, y todas ellas presuponen los beneficios mutuos de estas interacciones entre las comunidades locales y los recursos naturales (Berkes, 2001; Toledo, 2001, 2005; MMBT., 2004; Maffi, 2005; Pretty et al., 2008; Merino y Martínez, 2013). La integración efectiva de los pobladores locales en el uso, manejo y conservación de los recursos naturales en las Áreas Naturales Protegidas de México, los sitios más biodiversos del país es la única garantía para el desarrollo sustentable dada la condición de ruralidad, que se evidenciada en este estudio. 


\section{Consideraciones finales y perspectivas}

El papel de las poblaciones locales, especialmente los campesinos y más enfáticamente los indígenas, es esencial y requerido para el manejo y conservación de la naturaleza (RodríguezQuiroz, y Álvaro Bracamonte, 2008). Al respecto MMBT (2004), considera que de la gran diversidad de prácticas tradicionales, en diferentes lugares naturales alrededor del mundo; es posible identificar algunas características comunes a numerosas sociedades en su utilización de la biodiversidad: a) tienden a basarse en principios de reciprocidad; b) tienden a ser holísticas, percibiendo al entorno natural en su compleja trama de sistemas ecológicos que interactúan entre sí y del cual la comunidad es un componente más; c) generalmente tienen un vínculo estrecho con la identidad cultural y la autodeterminación local. Para algunos pueblos, las características del paisaje son parte integral de la forma en que reproducen su cultura.

Hablar de patrimonio natural en términos de manejo de los recursos naturales, encierra pues cierta forma de valorar, preservar y explotar la naturaleza; se trata de valores y categorías que no son universales (MMBT., 2004). Las personas de cada lugar tienen su manera propia de categorizar, valorar y usar su entorno natural, de hacer sus propias interpretaciones (Geertz, 1994), de forjarse su propia cultura e identidad. La propuesta teórica Axiomática de Toledo (2007), incluye propiciar la construcción de las Regiones Bioculturales de Conservación y Desarrollo con procesos de autonomías y autodeterminación. Lo cual coincide con lo descrito en Lagunas-Vázques et al. (2013): es menester la implementación de acciones de co-manejo, gobernanza local y derechos consuetudinarios partiendo de las conceptualizaciones teórico-prácticas más actuales, sobre desarrollo loca comunal, desarrollo sostenible, desarrollo humano y bienestar humano.

De acuerdo a Boege (2009) es importante reconocer que en México, así como la biodiversidad en general, incluyendo la cultural, los recursos fitogenéticos y los agroecosistemas indígenas en particular se encuentran amenazados. Dentro de las perspectivas de conservación propuestas por Boeger (2009), las estrategias de conservación de la biodiversidad in situ figuran como de primera importancia. Así como una serie de recomendaciones para integrar a los pueblos indígenas y al patrimonio biocultural de México en pro de la naturaleza (Boeger, 2009).

Este trabajo destaca la eminente ruralidad que existe en las ANP de México, así como la descripción de las principales actividades socioeconómicas y principales problemáticas 
sociambientales que son más comunes y generalizadas en ellas. Estas tres aportaciones conducen a la conclusión de que la resolución de tales problemáticas y el incremento de los niveles de vida de los pobladores locales de las ANP de México deben de transitar por soluciones locales de desarrollo rural sustentable.

\section{Agradecimientos}

Este trabajo fue desarrollado con el apoyo económico de los Proyectos 251919 de CONACYT Ciencia Básica y del Proyecto CONACYT-Redes Temáticas 269540. Este trabajo es parte de los resultados de las actividades de la Red Nacional Áreas Naturales Protegidas del CONACYT (RENANP). A la Dra. Alba Eritrea Gámez Vázquez Profesora-investigadora Departamento Académico de Economía Área de Conocimiento de Ciencias Sociales y Humanidades. Universidad Autónoma de Baja California Sur, por su lectura al documento y sus acertadas observaciones. Se agradecen además las acertadas observaciones de dos personas que revisaron el texto de forma anónima. Los Autores agradecemos al Lic. Gerardo Hernández el diseño gráfico editorial y a la Ms.C. Diana Dorantes la revisión del Idioma Inglés del Abstract.

\section{Literatura citada}

Alcorn, J. B. 1994. Noble savage or noble state? Northern myths and southern realities in biodiversity conservation. Etnoecológica, 2(3), 7-19.

Berkes, F., Colding, J., Folke, C. 2000. Rediscovery of traditional ecological knowledge as adaptive management. Ecological Applications. 10: 1251-1262.

Berkes, F. 2001. Managing small-scale fisheries: alternative directions and methods. IDRC. USA. 320 pp.

Bennett N. y P. Dearden. 2014a. Why local people do not support conservation: Community perceptions of marine protected area livelihood impacts, governance and management in Thailand. Marine Policy, Volume 44. Pages 107-116.

Bennett N., and P. Dearden, 2014b. From measuring outcomes to providing inputs: Governance, management, and local development for more effective marine protected areas. Marine Policy, Volume 50, Part A. Pages 96-110.

Bezaury-Creel, J., D. Gutiérrez Carbonell et al. 2009. Áreas naturales protegidas y desarrollo social 
en México, en Capital natural de México, vol. II: Estado de conservación y tendencias de cambio. Conabio, México, pp. 385-431.

Bobadilla M., Alvarez-Borrego S., Avila-Foucat S., Lara-Valencia F., y I. Espejel. 2011. Evolution of environmental policy instruments implemented for the protection of totoaba and the vaquita porpoise in the Upper Gulf of California. Environmental Science \& Policy, Volume 14, Issue 8, Pages 998-1007

Boege E. 2008 El patrimonio biocultural de los pueblos indígenas de México. INAH, CDI, México. Consultado: 25-02-2016. En: http://www.cdi.gob.mx/biodiversidad/ biodiversidad_0_ preliminares_1-31_eckart_boege.pdf

Boege, E. 2009. El reto de la conservación de la biodiversidad en los territorios de los pueblos indígenas, En: Dirzo, R., Gonzalez, R., \& March, I. J. Capital natural de México, vol. II: Estado de conservación y tendencias de cambio. (pp. 603-649). Conabio, México. 819 pp.

Chambers, R. y G. Conway. 1991. Sustainable rural livelihoods: Practical concepts for the $21^{\text {st }}$ century. IDS Discussion paper 296. UK. 33p.

Comisión Nacional de Áreas Naturales Protegidas CONANP. 2016. Áreas Naturales Decretadas. Consultado: 05-01-2016. En: http://www.conanp.gob.mx/que_hacemos/

Davidson-Hunt, I.J., Suich, H., Meijer, S.S. and Olsen, N. (eds.). 2016. People in Nature: Valuing the diversity of interrelationships between people and nature. Gland, Switzerland: IUCN. Gland, Switzerland: IUCN. 108pp.

Dirven, M. 2007. Pobreza rural y políticas de desarrollo: avances hacia los objetivos de desarrollo del Milenio y retrocesos de la agricultura de pequeña escala. CEPAL

Durand L. y J. Jiménez. 2010. Sobre áreas naturales protegidas y la construcción de no-lugares. Notas para México. Revista Lider Vol. 16 Año 122010 pp. 59-72

García-Bartolomé J.M. 1991. Servicios de Estudios del Ministerio de Agricultura Pesca y Alimentación. Política y Sociedad, 8 (1991), Madrid. 87-94 pp.

Geertz, C. 1994. Conocimiento local. Editorial Paidós. España.Instituto Nacional de Estadística y Geografía (INEGI). 2016. Metodologías para las encuestas a hogares. Consultado: 16/08/2016. En: http://www.inegi.org.mx/ est/contenidos

/espanol/metodologias/encuestas/hogares/Cae_ene.pdf

Lagunas-Vazques, M., Beltrán-Morales, L. F., Santiago-León, F. y A. Ortega-Rubio. 2013. Indicadores 
sociales: desarrollo humano en la reserva de la biosfera Sierra La Laguna. En: M. Lagunas-Vázques, L. F. Beltrán-Morales y A. Ortega-Rubio. (Editores). Diagnóstico y análisis de los aspectos sociales y económicos en la reserva de la biosfera Sierra La Laguna, Baja California Sur, México. (pp. 303-322). Centro de Investigaciones Biológicas del Noroeste S.C. La Paz, B.C.S., México. 475 pp.

Maffi, L. 2005. Linguistic, cultural, and biological diversity. Annu. Rev. Anthropol., 34, 599-617.

Mascia, M. B., Brosius, J. P., Dobson, T. A., Forbes, B. C., Horowitz, L., McKean, M. A., \& Turner, N. J. (2003). Conservation and the social sciences. Conservation biology, 17(3), 649-650.

Movimiento Mundial por los Bosques Tropicales (MMBT). 2004. Áreas Protegidas:

¿Protegidas contra quién? Uruguay. MMBT. 432 pp.

Adams B., Berkes F., Ferreira de Athayde S., Dudley N., Hunn E., Maffi L., Milton K., Rapport D., Robbins P., Samson C., Sterling E., Stolton S., Takeuchi K., Tsing A., Vintinner E. and S. Pilgrim. 2008. ¿How Do Biodiversity and Culture Intersect? Plenary paper for Conference "Sustaining Cultural and Biological Diversity In a Rapidly Changing World: Lessons for Global Policy". Organized by American Museum of Natural History's Center for Biodiversity and Conservation, IUCN-The World Conservation Union/Theme on Culture and Conservation, and Terralingua. April 2-5th 2008

Ostrom, E. 2001. Protecting the Commons: A Framework for Resource Management in the Americas.

J. Burger, R. B. Norgaard, D. Policansky y B. Goldstein (Eds.). Island Press. Washington, DC. Rábago, N. L. B., y Revah, L. O. 2014. El ecoturismo: ¿una nueva modalidad del turismo de masas? Economía Sociedad y Territorio.

Reyes, J.A., J.P. Gómez, R.O. Muis, R. Zavala, G.A. Ríos y O. Villalobos. 2012. Atlas de Propiedad Social y Servicios Ambientales en México. Instituto Interamericano de Cooperación para la Agricultura. Cooperación Técnica Registro Agrario Nacional - Instituto Interamericano de Cooperación para la Agricultura. México. 157 pp.

Rodríguez-Quiroz y A. Bracamonte. 2008. Pertinencia de las ANP como política de conservación y mejoramiento dela calidad de vida. Análisis de percepción en la Reserva de la Biosfera del Alto Golfo de California y Delta del Río Colorado. Estudios Sociales, 16(32): 143-177.

Toledo, V. M. 2001. Biodiversity and indigenous peoples. En: Levin, S. (ed) Encyclopedia of Biodiversity. Academic Press: 1181-1197. 
Toledo V., 2005. La ecología rural. Ciencia y Desarrollo (México) 174: 36-43.

Toledo, V. M. 2005. Repensar la conservación: ¿áreas naturales protegidas o estrategia bioregional? Gaceta Ecológica, núm. 77, octubre-diciembre, 2005, pp. 67-83

Yang H., Rhett H., Zhuang-Fang Y., Goodale E., Ming-Xu Z., and X. Jian-Chu. 2015. “Changing Perceptions of Forest Value and Attitudes toward Management of a Recently Established Nature Reserve: A Case Study in Southwest China. Forests 6, no. 9: 3136-3164.

Yang H., Rhett H., Zhuang-Fang Y., Goodale E., Ming-Xu Z., y X. Jian-Chu. 2015. “Changing Perceptions of Forest Value and Attitudes toward Management of a Recently Established Nature Reserve: A Case Study in Southwest China. Forests 6, no. 9: 3136-3164.

World Commission on Protected Areas (WCPA). 2016. What is a protected area? Consultado: 15/08/2016. En: https://www.iucn.org/theme/protected-areas/about World Wildlife Fund (WWF). 2000. Indigenous and Traditional Peoples of the World and Ecoregion Conservation. Suiza. 120 pp.

\section{Cita}

Lagunas-Vázques M., L. F. Beltrán-Morales, M. Bobadilla-Jiménez y A. Ortega-Rubio. 2016. Población humana, actividades socioeconómicas y problemáticas socioambientales de las Áreas Naturales Protegidas (ANP) de México. Áreas Naturales Protegidas Scripta. Vol.II (2): 67-89. https://doi.org/10.18242/anpscripta.2016.02.02.02.0004

Sometido: 24 de Agosto de 2016

Revisado: 21 de Septiembre de 2016

Aceptado: 17 de Octubre de 2016

Editora asociada: Dra. Alba Eritrea Gámez Vázquez

Idioma Inglés Abstract: Ms.C. Diana Dorantes

Diseño gráfico editorial: Lic. Gerardo Hernández 
Anexo 1. Población total de cada ANP, que cuentan con datos de habitantes y porcentaje de población indígena (\%) en México. Fuente: Elaboración propia con datos tomados de la CONANP (2016)

\begin{tabular}{|c|c|c|c|}
\hline Categoria & Nombre & Población & P. Indígena \\
\hline RB & $\begin{array}{l}\text { Alto Golfo de California y Delta del } \\
\text { Rio Colorado }\end{array}$ & 5141 & $101(2 \%)$ \\
\hline RB & Barranca de Metztitlán & 26533 & $1533(5.8 \%)$ \\
\hline RB & Calakmul & 2707 & $1594(60 \%)$ \\
\hline RB & Chamela-Cuixmala & 26 & \\
\hline RB & El Pinacate y Gran Desierto de Altat & 49 & $1(2 \%)$ \\
\hline RB & El Triunfo & 10747 & $971(9 \%)$ \\
\hline RB & El Vizcaíno & 51211 & $5340(10.4 \%)$ \\
\hline $\mathrm{RB}$ & Isla Guadalupe & 92 & $4(4.3 \%)$ \\
\hline $\mathrm{RB}$ & Islas Marías & 2788 & $131(4.7 \%)$ \\
\hline $\mathrm{RB}$ & Janos & 3411 & $475(14 \%)$ \\
\hline $\mathrm{RB}$ & La Encrucijada & 16688 & $71(0.4 \%)$ \\
\hline $\mathrm{RB}$ & La Sepultura & 8468 & $1032(12.2 \%)$ \\
\hline RB & Los Tuxtlas & 28611 & $7824(27.3 \%)$ \\
\hline $\mathrm{RB}$ & Mapimí & 401 & \\
\hline $\mathrm{RB}$ & Mariposa Monarca & 27346 & $644(2.4 \%)$ \\
\hline $\mathrm{RB}$ & Marismas Nacionales Nayarit & 1474 & \\
\hline $\mathrm{RB}$ & Montes Azules & 19921 & $16204(81.3 \%)$ \\
\hline $\mathrm{RB}$ & Pantanos de Centla & 24536 & $1991(8.1 \%)$ \\
\hline $\mathrm{RB}$ & Ría Celestún & 7580 & $1455(19.2 \%)$ \\
\hline RB & Ría Lagartos & 6782 & $1825(27 \%)$ \\
\hline $\mathrm{RB}$ & Selva El Ocote & 8017 & $4922(61.4 \%)$ \\
\hline $\mathrm{RB}$ & Sian Ka'an & 634 & $202(32 \%)$ \\
\hline $\mathrm{RB}$ & Sierra de Huautla & 3922 & $27(0.7 \%)$ \\
\hline $\mathrm{RB}$ & Sierra de Manantlán & 8416 & $87(1 \%)$ \\
\hline $\mathrm{RB}$ & Sierra del Abra Tanchipa & 3 & \\
\hline $\mathrm{RB}$ & Sierra Gorda & 98811 & $1193(1.2 \%)$ \\
\hline $\mathrm{RB}$ & Sierra Gorda de Guanajuato & 24385 & $117(0.5 \%)$ \\
\hline $\mathrm{RB}$ & Sierra La Laguna & 409 & \\
\hline RB & Tehuacán-Cuicat lán & 36471 & $16934(46.4 \%)$ \\
\hline $\mathrm{RB}$ & Volcán Tacaná & 738 & $196(26.6 \%)$ \\
\hline $\mathrm{RB}$ & Zicuirán-Infiernillo & 11944 & $43(0.4 \%)$ \\
\hline $\mathrm{RB}$ & $\begin{array}{l}\text { Zona marina Bahía de los Ángeles, } \\
\text { canales de Ballenas y Salsipuedes }\end{array}$ & 1 & \\
\hline $\mathrm{PN}$ & Arrecife de Puerto Morelos & 2 & \\
\hline $\mathrm{PN}$ & Bahía de Loreto & 4 & \\
\hline $\mathrm{PN}$ & Bosencheve & 16351 & $6999(42.8 \%)$ \\
\hline $\mathrm{PN}$ & Cañón del Río Blanco & 3297999 & $26900(0.8 \%)$ \\
\hline $\mathrm{PN}$ & Cañón del Sumidero & 52672 & $4130(7.8 \%)$ \\
\hline $\mathrm{PN}$ & Cascada de Bassaseachic & 94 & $7(7.4 \%)$ \\
\hline \multirow{2}{*}{$\mathrm{PN}$} & Cofre de Perote o & & \\
\hline & Nauhcampatépetl & 4229 & $27(0.6 \%)$ \\
\hline $\mathrm{PN}$ & Cumbres de Majalca & 35 & $1(3 \%)$ \\
\hline $\mathrm{PN}$ & Cumbres de Monterrey & 2848 & $42(1.5 \%)$ \\
\hline $\mathrm{PN}$ & Desierto del Carmen o de Nixcongo & 1032 & \\
\hline $\mathrm{PN}$ & El Chico & 505 & $6(1.2 \%)$ \\
\hline $\mathrm{PN}$ & El Potosí & 81 & \\
\hline
\end{tabular}




\begin{tabular}{|c|c|c|c|}
\hline Categoria & Nombre & Población & P. Indígena \\
\hline $\mathrm{PN}$ & El Tepozteco & 42904 & $4789(11.2 \%)$ \\
\hline $\mathrm{PN}$ & El Veladero & 575 & $32(5.6 \%)$ \\
\hline $\mathrm{PN}$ & General Juan Álvarez & 29 & \\
\hline $\mathrm{PN}$ & Gogorrón & 26872 & $108(0.4 \%)$ \\
\hline $\mathrm{PN}$ & Grutas de Cacahuamilpa & 1359 & $6(0.4 \%)$ \\
\hline $\mathrm{PN}$ & Huatulco & 109 & $20(18.3 \%)$ \\
\hline $\mathrm{PN}$ & Insurgente José María Morelos & 1755 & $25(1.4 \%)$ \\
\hline $\mathrm{PN}$ & Insurgente Miguel Hidalgo y Costilla & 2666 & $141(5.3 \%)$ \\
\hline $\mathrm{PN}$ & Iztaccíhuatl-Popocatépetl & 244 & \\
\hline $\mathrm{PN}$ & La Montaña Malinche o Matlalcuéyatl & 44097 & $33562(76 \%)$ \\
\hline $\mathrm{PN}$ & Lagunas de Chacahua & 2766 & $187(6.8 \%)$ \\
\hline $\mathrm{PN}$ & Lagunas de Montebello & 1587 & $15(1 \%)$ \\
\hline $\mathrm{PN}$ & Los Mármoles & 6043 & $1052(17.4 \%)$ \\
\hline $\mathrm{PN}$ & Palenque & 186 & $42(23 \%)$ \\
\hline $\mathrm{PN}$ & Sacromonte & 18 & \\
\hline $\mathrm{PN}$ & Tulum & 13 & $3(23 \%)$ \\
\hline $\mathrm{PN}$ & Xicoténcatl & 37221 & $1622(4.4 \%)$ \\
\hline $\mathrm{MN}$ & Bonampak & 11 & $11(100 \%)$ \\
\hline $\mathrm{MN}$ & Cerro de la Silla & 8 & \\
\hline $\mathrm{MN}$ & Yagul & 149 & $52(35 \%)$ \\
\hline APRN & $\begin{array}{l}\text { Cuenca Alimentadora del Distrito } \\
\text { Nacional de Riego Pabellón } \\
\text { Cuenca Alimentadora del Distrito }\end{array}$ & 1025 & $2(0.2 \%)$ \\
\hline APRN & Nacional de Riego Don Martín & 2665 & $12(0.5 \%)$ \\
\hline APRN & $\begin{array}{l}\text { Cuenca Alimentadora del Distrito } \\
\text { Nacional de Riego } 0 \text { Bajo Río San Juan }\end{array}$ & 5509 & $16(0.3 \%)$ \\
\hline APRN & $\begin{array}{l}\text { Cuenca Alimentadora del Distrito } \\
\text { Nacional de Riego Estado de Nayarit }\end{array}$ & 52498 & $25768(50 \%)$ \\
\hline & $\begin{array}{l}\text { Zona de Protección Forestal en los } \\
\text { terrenos que se encuentran en los } \\
\text { municipios de La Concordia, Angel }\end{array}$ & & \\
\hline APRN & Albino Corzo, Villa Flores y Jiquipilas & 10095 & $3059(30 \%)$ \\
\hline APRN & $\begin{array}{l}\text { Zona Protectora Forestal los terrenos } \\
\text { constitutivos de las cuencas de los ríos } \\
\text { Valle de Bravo, Malacatepec, Tilostoc } \\
\text { y Temascaltepec }\end{array}$ & 198708 & $22424(11 \%)$ \\
\hline APRN & $\begin{array}{l}\text { Zona Protectora Forestal Vedada } \\
\text { Cuenca Hidrográfica del Río Necaxa }\end{array}$ & 137509 & $58530(43 \%)$ \\
\hline APFF & Bala'an K'aax & 158 & $105(66.5 \%)$ \\
\hline APFF & Balandra & 2 & \\
\hline APFF & Cabo San Lucas & 33 & \\
\hline APFF & Campo Verde & 97 & $1(1 \%)$ \\
\hline APFF & Cañón de Santa Elena & 1454 & $5(0.3 \%)$ \\
\hline APFF & Cañón del Usumacinta & 8156 & $3158(38.7 \%)$ \\
\hline APFF & Cascada de Agua Azul & 1872 & $1815(97 \%)$ \\
\hline APFF & Ciénegas del Lerma & 574 & $9(1.6 \%)$ \\
\hline
\end{tabular}




\begin{tabular}{|c|c|c|c|}
\hline Categoria & Nombre & Población & P. In dígena \\
\hline APFF & Corredor Biológico Chichinautzi & 31653 & $1354(4.3 \%)$ \\
\hline APFF & Cuatrociénegas & 92 & \\
\hline APFF & El Jabalí & 60 & \\
\hline APFF & Islas del Golfo de California & 3214 & $131(4 \%)$ \\
\hline APFF & La porción norte y la franja cost & 17 & $16(94 \%)$ \\
\hline APFF & La Primavera & 97 & $11(11 \%)$ \\
\hline APFF & Laguna de Términos & 205487 & $5584(2.7 \%)$ \\
\hline APFF & Laguna Madre y Delta del Río Bi & 17199 & $746(4.3 \%)$ \\
\hline APFF & Maderas del Carmen & 45 & \\
\hline APFF & Médanos de Samalayuca & 1578 & $27(1.7 \%)$ \\
\hline APFF & Meseta de Cacaxtla & 1162 & \\
\hline APFF & Metzabok & 96 & $96(100 \%)$ \\
\hline APFF & Nahá & 204 & $204(100 \%)$ \\
\hline APFF & Nevado de Toluca & 5297 & $93(1.8 \%)$ \\
\hline APFF & Ocampo & 1073 & \\
\hline APFF & Otoch Ma'ax Yetel Kooh & 151 & $151(100 \%)$ \\
\hline APFF & Papigochic & 6907 & $712(10.3 \%)$ \\
\hline APFF & Pico de Tancítaro & 1289 & $49(3.8 \%)$ \\
\hline APFF & Sierra de Álamos-Río Cuchujaqui & 542 & $4(0.7 \%)$ \\
\hline APFF & Sierra de Álvarez & 1110 & \\
\hline APFF & Tutuaca & 4168 & $991(23.8 \%)$ \\
\hline APFF & Valle de los Cirios & 1993 & $71(3.6 \%)$ \\
\hline APFF & Yum Balam & 2957 & $636(21.5 \%)$ \\
\hline APFF & Cerro Mohinora & 298737 & $15969(5.3 \%)$ \\
\hline SAN & Islas La Pajarera, Cocinas, Mam & 15 & \\
\hline SAN & Playa de Puerto Arista & 69 & \\
\hline
\end{tabular}


Anexo 2. Principales actividades socioeconómicas y problemáticas socioambientales por frecuencia o veces que se presentan en las fichas técnicas de cada una de las ANP. Fuente: Elaboración propia con datos tomados de la CONANP (2016)

\section{problemática/veces que se repite}

Contaminación agua y suelo Incendios forestales

Tráfico ilegal de especies

Tala clandestina

Malas prácticas turísticas Introducción de especies exóticas

Cacería y/o pesca, Ilegal/furtiva

Erosión

Sobre explotación de RN Daño poblaciones silvestres

Mal manejo residuos

Invasión de tierras

Fenomenos naturales

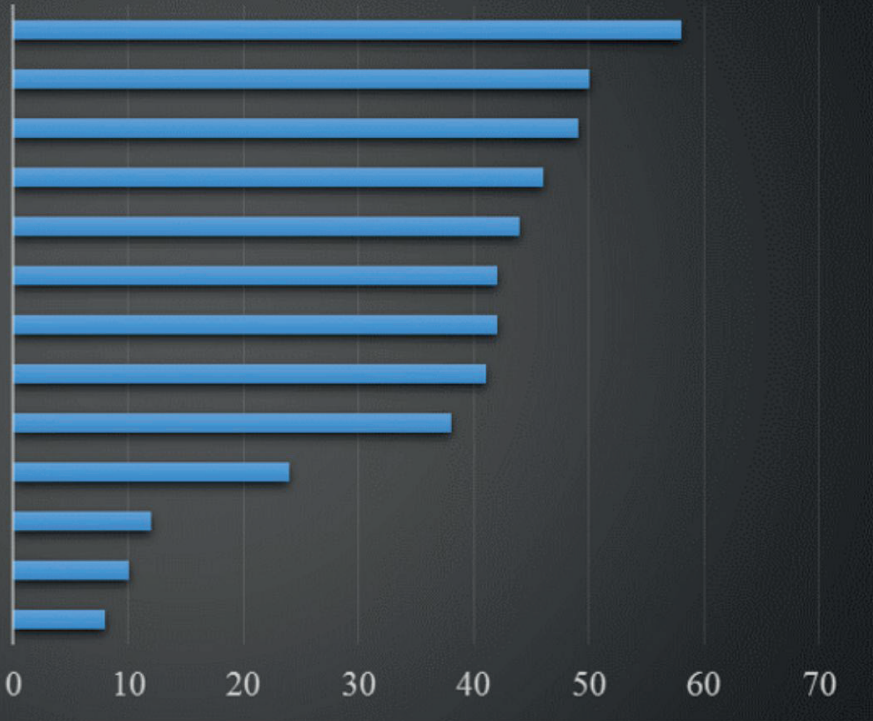

\section{actividad/veces que se repite}

Nuevos asentamiento y desarrollo urbano

Ganaderia extensiva

Expansión Agrícolas/pecuarias

Cambio de uso de suelo

Infraestructura turística alto impacto Explotación forestal no planificada Extracción de materiales y mineria Construcción de autopistas y carreteras

Extracción fauna/cacería/pesca

Aprovechamiento de RN

Desarrollo industrial

Turismo/recreación Explotación petrolera

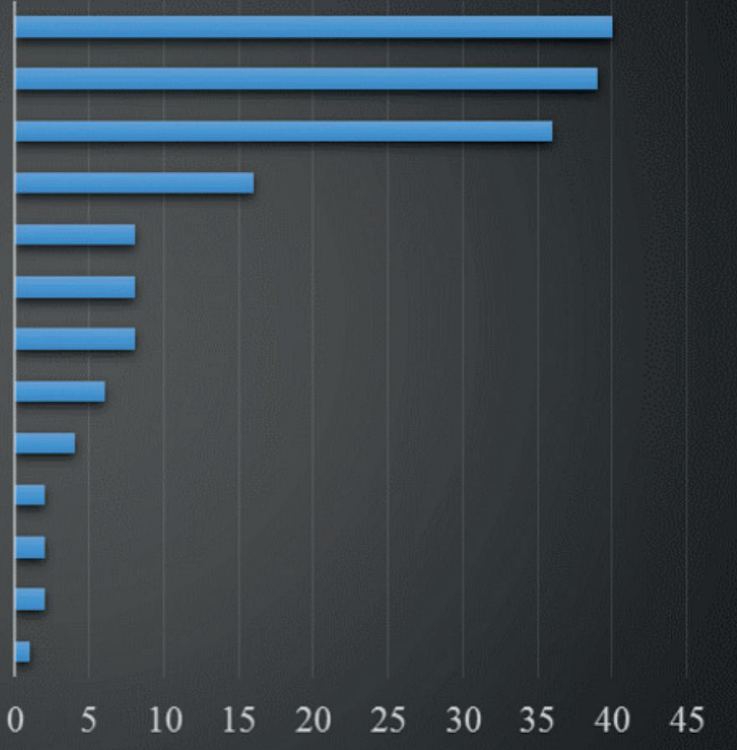

